\title{
BUTT, John, Music education and the art of performance in the German Baroque
}

Patrice Veit

\section{OpenEdition}

\section{Journals}

Édition électronique

URL : http://journals.openedition.org/ifha/1402

DOI : $10.4000 /$ ifha. 1402

ISSN : 2198-8943

\section{Éditeur}

IFRA - Institut franco-allemand (sciences historiques et sociales)

Référence électronique

Patrice Veit, «BUTT, John, Music education and the art of performance in the German Baroque », Revue de I'IFHA [En ligne], Date de recension, mis en ligne le 01 janvier 1999, consulté le 22 septembre 2020. URL : http://journals.openedition.org/ifha/1402 ; DOI : https://doi.org/10.4000/ifha.1402

Ce document a été généré automatiquement le 22 septembre 2020

(CIFHA 


\title{
BUTT, John, Music education and the art of performance in the German Baroque
}

\author{
Patrice Veit
}

1 Issu de deux colloques tenus à New-York et à Chicago, le volume dirigé par J.K. explore la culture musicale de l'espace germanophone entre 1450 et 1600 . Les quatorze contributions rassemblées et organisées autour des trois parties figurant dans le titre tentent d'en mesurer la place et l'influence dans l'espace européen. S'y trouve ainsi analysée la fonction de modèle joué par l'espace germanophone non seulement dans le patronage musical à l'exemple de la cour de l'Empereur Rodolphe II, mais aussi dans l'établissement du chant d'assemblée dans les églises et dans le développement de l'impression musicale. Au-delà, l'ouvrage démontre le rôle fondamental joué par les musiciens de langue allemande dans le développement de la musique instrumentale à l'époque de la Renaissance, que ce soit par Conrad Paumann et Arnold Schlick pour la musique d'orgue ou Hans Newsidler pour celle de luth, et le rayonnement de nombreux instrumentistes allemands dans les diverses cours européennes. Les études détaillées apportent en outre de précieux éclaircissements sur la pratique musicale dans les cours, dans les universités, dans les écoles latines, mais aussi dans les cercles humanistes à l'exemple de Glaréan et des Amerbach à Bâle ainsi que dans les intérieurs $\mathrm{du}$ patriciat urbain comme le révèle la riche bibliothèque musicale de Georg Knoff à Danzig à la fin du XVIe s. Les différentes contributions mettent en évidence 1 'importance musicale de l'époque de Gutenberg, de Dürer et de Luther: d'une part, cette période marque l'apogée de la musique polyphonique allemande; d'autre part, elle voit naître un genre, le Tenorlied, qui assure à l'Empire une place éminente au sein des nations musicales en Europe et auquel contribuent quelques-uns des musiciens les plus prestigieux du XVIe s. comme Paul Hofhaimer, Heinrich Isaac ou Ludwig Senfl; enfin, elle se caractérise par une profonde transformation du statut social de la musique, grâce en partie à l'humanisme et à la Réforme. 
2 Prolongeant chronologiquement l'ouvrage précédent, l'étude particulièrement stimulante du musicologue de l'Université de Berkeley, John BUTT, s'intéresse à reconstruire l'art de l'interprétation en Allemagne à l'époque baroque, en prenant pour cadre institutionnel les églises et les écoles des territoires luthériens pour y étudier notamment le rôle de la pratique musicale dans l'éducation. J.B. justifie son choix par l'extraordinaire réussite des musiciens allemands protestants de l'époque baroque, mais surtout par la place faite à la pratique musicale dans le système éducatif des écoles latines luthériennes. En accordant une fonction particulière au chant et à la musique chargés de diffuser la Parole divine, la Réforme luthérienne faisait en même temps de l'église et de l'école les deux centres de la culture musicale au XVIe s. L'importance donnée à la musique et l'accent mis sur le lien texte - musique expliquent aussi la riche production, du côté luthérien, de traités et de manuels à but pratique, rédigés aussi bien par des pasteurs, des cantors que des Kapellmeister. L'intérêt de la recherche de J.B. repose en particulier sur l'exploitation systématique de plus de 230 préfaces, traités et manuels d'instruction utilisés dans les écoles latines luthériennes au cours de la période comprise entre 1530 et 1800 , depuis les Rudimenta musicae de N. Listenius (1533) jusqu'à la Kurze und erleichterte Anweisung zum Singen de J. A. Hiller (1792). L'examen de ces divers textes, complété par celui des ordonnances ecclésiastiques et scolaires, éclaire la fonction et le statut de la musique à l'école et à l'église, considérée comme un art fonctionnel (musica practica); il permet en outre de mieux comprendre la pratique musicale et, ce faisant, d'approcher les théories des interprètes de l'époque ainsi que la manière dont ceux-ci ont pu interpréter les œuvres sacrées d'un Praetorius, d'un Schütz, d'un Buxtehude ou d'un Bach. Après une présentation de l'établissement de la pratique musicale luthérienne au XVIe s. ainsi que de l'évolution de la musique pratique dans le système éducatif au XVIIe s., l'ouvrage de J.B. se penche sur la forme, le style et le contenu des différents traités, avant d'aborder de manière approfondie le développement de l'interprétation pratique au XVIIe s. ainsi que les questions relatives à l'expression et à l'interprétation dans l'Allemagne de l'époque baroque. Selon J.B., l'apparition de l'interprétation n'est pas seulement le fait du nouveau style italien introduit en Allemagne en particulier par Michael Praetorius mais représente un phénomène consubstantiel à l'esprit du luthéranisme qui envisage la musique pratique comme un discours théologique parlant aux sens. Les différents textes aux liens étroits les uns par rapport aux autres, constituent un corpus théorique reflétant les vues des contemporains sur l'interprétation pratique et sur son rôle dans la vie musicale et dans l'éducation. L'interprétation concerne avant tout le chant, les problèmes d'articulation, de registres, de prononciation, de distribution des voix, d'ornementation mais aussi l'apprentissage des instruments. Alors que le XVIIe s. et la première moitié du XVIIIe s. représentent une période de plein essor pour la musique à l'église et à l'école, on constate à partir du milieu du XVIIIe s. un déclin de la pratique du chant dans les écoles, phénomène bien connu par exemple pour l'École Saint-Thomas de Leipzig dans les dernières années du cantorat de Bach. Ce déclin tient non seulement à la stagnation de nombreux cantorats mais plus généralement à un changement d'attitudes concernant la fonction et le statut de la musique, envisagée avec l'Aufklärung comme un art esthétique et non plus fonctionnel. Cette étude bien menée est en outre très utile par la richesse et le choix des exemples cités à partir des sources originales. 